\title{
A Novel Bandwidth Efficient PTS Scheme for Peak Power Reduction in OFDM Systems
}

\author{
Alok Joshi and Samriti Kalia \\ Department of ECE \\ Jaypee Institute of Information Technology, Noida, India
}

\begin{abstract}
Orthogonal Frequency Division Multiplexing (OFDM) OFDM is a multicarrier transmission technique that has wide ranging applications in wireless communications. It is the basis of many prominent IEEE standards, pre-4G and 4G technologies. It improves bandwidth efficiency and also withstands multipath propagation to provide seamless communication. However, it experiences the issue of high peak-to- average- power ratio (PAPR) causing signal degradation. The Partial Transmit Sequence (PTS) is most efficient technique to reduce high PAPR, but it too suffers from a few problems - one of which is the need for transmission of extra (overhead) bits, known as side information, to successfully recover the OFDM symbol at the receiver end, but this leads to deterioration of spectrum efficiency of the system. The paper proposes a novel method using constellation mapping with some simple mathematical constraints that could help to resolve the problem of side information in PTS thus saving the bandwidth.
\end{abstract}

Keywords: OFDM, PTS, Side information, PAPR

\section{Introduction}

Orthogonal Frequency Division Multiplexing (OFDM) [1, 2] is now become a standard spectrum efficient method for high data rate applications. Since it uses narrowband channels which make it immune to frequency selective fading and inter-symbol interference (ISI), using cyclic prefix avoids any adverse affect of delay spread such as inter-carrier interference (ICI) $[3]$.

Even though there so many advantages, OFDM also has some limitations such as a large Peak to Average Power Ratio (PAPR). High peak in the time domain envelopes are caused due to inverse fast Fourier transform (IFFT) taking place at the transmitter. IFFT sums up the modulated sub-carriers which generates high peaks when number of subcarriers gets aligned in phase. OFDM signal with high PAPR causes harmonic distortion and out of band radiations when experiences nonlinearity such as high power amplifier. Reducing PAPR ensures that HPA works in its linear region.

Various methods [4] are discussed in the literature for the sake of PAPR reduction such as clipping [5] where higher peaks are clipped but due to non linearity of operation generates distortion which can be reduced by clipping-filtering [6,7], peak windowing method [8] uses a windowing function to hard limit the peaks, companding [9] transforms are also used for PAPR reduction, peak cancellation [10] uses a waveform which is subtracted from OFDM signal from the areas where high peaks are observed. But all these methods reduce PAPR at but at higher bit error rate (BER).

Coding methods such as Golay codes [11], Reed-Muller codes [11], LDPC codes [12] and Turbo codes [13] are also used for PAPR reduction but not suitable for large constellation.

The other distortionless methods for PAPR reduction are Tone injection [14], Tone reservation [15], Active constellation extension [16]. These methods modify the constellation by adding additional points, but PAPR reduction costs in higher transmission power requirement. Selective mapping (SLM) [17,18,19] and Partial transmit sequence (PTS) $[20,21]$, both are multiple signal representation methods, where multiple phase combinations are used on data set to generate multiple OFDM signal representation for same data signal and the representation with lowest PAPR is used for final transmission.

Received: May $16^{\text {th }}, 2018$. Accepted: May $23^{\text {rd }}, 2020$ 
PTS is more in use due to lower computation complexity. In PTS data is subdivided in equal size independent sub-blocks, each sub-block is multiplied by a phase factor then added together to generate an OFDM signal, all possible combinations of phase sets are used to generate multiple OFDM representation and representation with lowest PAPR representation is used for transmission. But information about the phase sequence used is required for error free detection of OFDM symbol at the receiver, this information is called side information (SI), Transmission of SI effectively brings down the bandwidth efficiency as SI burdens the bandwidth.

There are schemes which offers detection without need of SI some of the popular schemes are discussed here.

In [22] to avoid SI transmission a predetermined set of pilot signals are inserted in each sub-block for easing out the SI detection, but due to maximum likelihood detection (ML) receiver complexity is high. In [23] cyclic shifting is used in place of multiplying the phase factors but again receiver complexity is high due to ML detection. In [24] a modified constellation for QAM is used for both SLM and PTS and at the receiver ML method is used for recovery of the signal. In [25] similar to [22] pilot carriers and ML method is used. In [26] single IFFT operation is performed with joint phase rotation and offset is used to reduce the complexity and complex ML method is used at the receiver. In [27] the author proposed a phase offset method for Alamouti MIMO transmitter with a complex receiver employing ML method. In [28] wavelet packet based OFDM is used to reduce complexity but uses embedded SI with complex receiver design. [29] too uses phase offset on rotating vectors and uses embedded SI with ML based receiver.

In this paper, the proposed method eliminates the need of side information for recovery of symbols in PTS without degrading PAPR reduction performance. The scheme uses constellation mapping and mathematical equations to identify the symbols sent by the receiver. The main idea is to map the symbols in the complex plane, then derive the relationships between the real and imaginary parts and finally form the mathematical equations (with the help of derived relationships) to recover the symbols at the receiver side. The method has been described in detail in a later Section.

\section{Peak to Average Power ratio and PTS Scheme}

IFFT sum used at the transmitter may results in to high peaks in the OFDM signal envelope.

An OFDM signal with $\mathrm{N}$ subcarrier for the input data can be represented as given in (1):

$$
\operatorname{PAPR}(x)=\frac{\max _{0<n<N-1}\left|x_{n}\right|^{2}}{E\left[\left|x_{n}\right|^{2}\right]} \quad n=0,1 \ldots . . N-1
$$

\section{Multiple signal representation method- PTS}

In partial transmit sequence method; the basic idea is to divide the input sequence into number of independent sub-blocks and to find the set of optimal rotating phase factors that give minimum value of PAPR on multiplying with different sub-blocks. The typical functional blocks of the PTS scheme is shown in Figure 1.

Input data sequence $M$ is divided into $U$ sub-blocks having same size equal to $N$.

$$
M=\sum_{u=1}^{U} M_{u}
$$




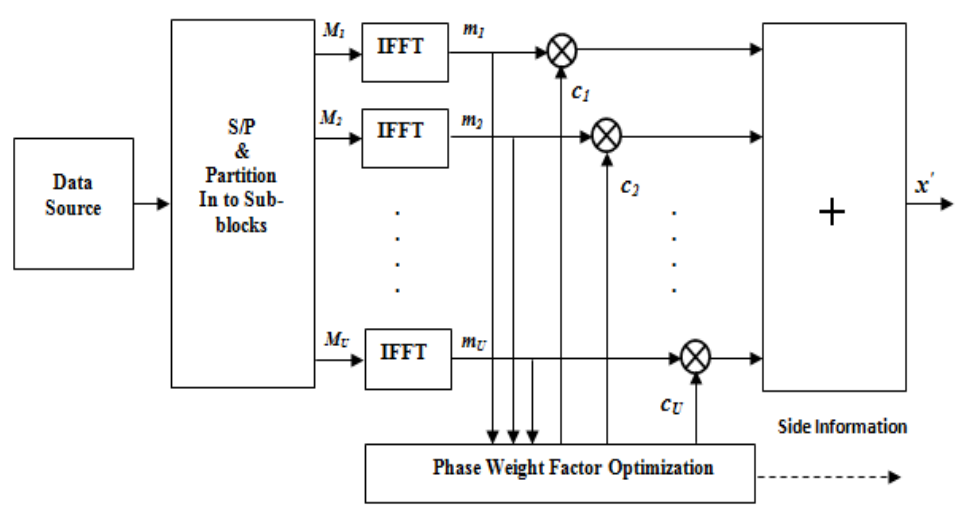

Figure 1. Building blocks of plain-PTS technique

Hence, each sub-block contains almost $N / U$ non-zero elements with zero padding in the rest of the sequence. The IFFT of each sub-block vector is multiplied by a phase sequence $c$ of magnitude as unity; the summation of these rotated sub-block vector is given as follows:

$$
x=\sum_{u=1}^{U} c_{u} m_{u}
$$

One appropriate optimum phase factor is selected from $c=\left[c_{1}, c_{2}, \ldots, c_{u}\right]$ which produces minimum PAPR. The combination is found by

$$
\begin{aligned}
\widetilde{c} & =\left[c_{1}, c_{2}, \ldots, c_{u}\right] \\
& =\underset{c_{1}, c_{2}, \ldots, c_{u}}{\arg \min }\left[\max _{0 \leq n \leq N-1}\left|\sum_{u=1}^{U} c_{u} m_{u}\right|\right]
\end{aligned}
$$

Using above equation we can find the optimum phase factor which optimizes the PAPR performance. The minimum PAPR signal transmitted is represented as

$$
\tilde{x}=\sum_{m=1}^{U} \widetilde{c}_{u} \cdot m_{u}
$$

The conventional PTS algorithm requires to send some side information about phase factors to the receiver in order to detect the received symbols correctly.

The redundant bits are equal to $\quad(U-1) \log _{2} O$

Where $O$ represents the possible phase weights i.e. $\{ \pm 1, \pm j\}$.

Transmission of side information degrades the spectral efficiency of the OFDM system.

For finding the optimal phase factors among the all possible set $\widetilde{c}=\left[c_{1}, c_{2}, \ldots, c_{u}\right]$, many computations will be required if this set is very large.

For example, for $O$ possible phase weights, then set phase set $c$ will have $O^{U}$ combinations and thus a total of $\left(U O^{m}\right)$ IFFT operations would be required. Increasing the values $U$ and $O$ would thus mean higher computational cost in implementing the algorithm. If first block is kept unchanged then phase combination will be $O^{U-1}$.

Apart from the IFFT operations each OFDM signal generated for a phase set combination will require N.K.O $O^{U-1 .}(U-1)$ complex addition and multiplications. Where $K$ is the oversampling factor 


\section{The Proposed PTS without side information}

The proposed method, as mentioned earlier, is based on mapping the symbols to certain predetermined points on the constellation plane and then deriving relationships between all the different points with respect to their real and imaginary parts. In this method, the receiver requires two symbols in order to detect them correctly without any side information. This scheme does not require any new building blocks at the transmitter side of PTS scheme. Thus no addition to hardware complexity at the transmitter. Only at the receiver with the help of a memory element and exploiting the relation between transmitted constellation the symbol detection takes place.

The method was initially proposed for a set of two phase factors, but can also work with a set of four phase factors with the help of minor adjustments. The working of the method with two phase factors and four phase factors are given in Part 1 and Part 2 respectively.

\section{A. The Proposed method with 2 phase weights}

1. Suppose we have two phase factors $(+1,-1)$ or $(+j,-j)$

2. Let the input data be mapped to certain points in the complex plane. Let these points be denoted by A, B, C and D respectively. These points (A, B, C and D) are chosen such that the relationship of absolute ratio of real and imaginary parts between two points is unique.

For example, let us say we have three points in the complex plane which are $a+j b, c+j d$ and $e+j f$. Then, $|a / c|$ should be equal to the absolute ratio of real parts of only one other mapped point i.e. either $|c / e|$ or $|a / e|$ and not both. Similarly for the imaginary parts, $|b / d|$ should be equal to the absolute ratio of imaginary parts of only one other point i.e. either $|d / f|$ or $|b / f|$ and not both. An example of mapping of points is shown in Figure 2. In this Figureure points $1+j, 2+3 j, 4+2 j, 5+4 j$ are mapped.

3. After mapping, we multiply the symbols by appropriate phase factors and then send the symbols one after the other.

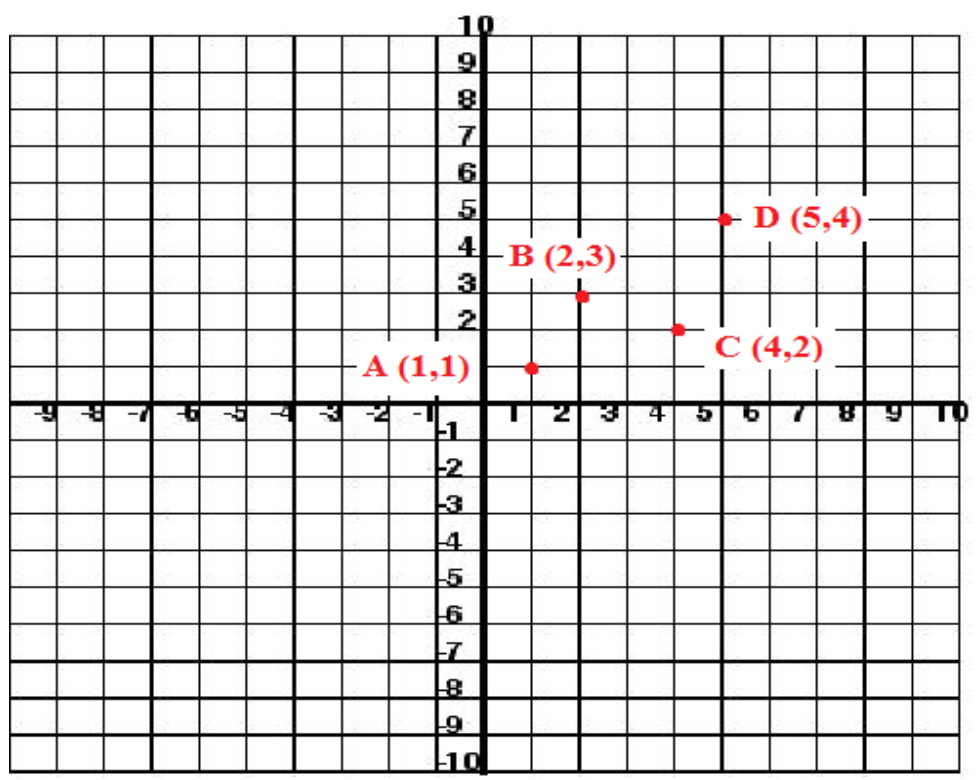

Figure 2. Mapping of data symbols 
4. The receiver, as soon as it receives two symbols, can detect them based on certain conditions (or equations) which are already present at the receiver. Depending on the points chosen for mapping (in this case $\mathrm{A}, \mathrm{B}, \mathrm{C}$ and $\mathrm{D}$ ), equations are formed and stored at the receiver accordingly and can be in the form of a look-up table.

Let us take, for example, points B and C, we can see that they have a unique relationship such that

$\operatorname{Re} .(C)=2 \times \operatorname{Re} .(B)$

Img. $(C)=(2 / 3) \times$ Img. $(C)$

Where Re. represents real and Img represents imaginary part.

This relationship is true only for points $\mathrm{B}$ and $\mathrm{C}$ and no other set of points. These simple relationships are then used to form the required equations.

Let us assume that two points received at the receiver are $\left(a_{1}, b_{1}\right)$ and $\left(a_{2}, b_{2}\right)$ where a represent the real parts and $b$ represent the imaginary parts of the received points respectively.

The equations for detecting points $\mathrm{A}, \mathrm{B}, \mathrm{C}$ and $\mathrm{D}$ are given in Table 1 .

5. At the receiver, we can find out which symbols were sent with the help of the corresponding equations as given in Table 1.

6. We can see that equations for A A, B B, C C, D D are same, so receiver will have a problem in Figuring out which of these symbols were transmitted. To solve this problem, we can use two methods:

- Method 1:

We will calculate Euclidean distance from the origin to the received point. With the help of this distance, we'll know which symbols among A A, B B, C C, D D were transmitted.

- Method 2:

We can have a memory element at the receiver side which stores the first two distinct symbols that it receives, and uses these symbols at a later time when it faces the above mentioned problem of detecting which symbols among A A, B B, C C, D D were sent by the transmitter.

Suppose if we send symbols A A C B one after the other, then the receiver would see that first two symbols are the same, (i.e. A A in this case), so it would store one of these two and wait for the next distinct symbol (i.e. $\mathrm{C}$ in this case), so in summary, it would store symbols $\mathrm{A}$ and $\mathrm{C}$ in this case.

To make things clear, a case is discussed below wherein the transmitter sends the symbols B B B A C D DA in succession.

a) Suppose the transmitter has two transmit the sequence B-B-B A C D D A.

b) First, it will transmit symbols $\mathrm{B}$ and $\mathrm{B}$, one after the other.

c) As soon as the first two symbols are received, the receiver uses the equations (from Table 1) to find out which symbols were sent.

d) In this case, the receiver finds out that the first two symbols are same, but faces a difficulty to determine which combination of symbols among A A, B B, C C or D D was sent.

e) It counters this problem by storing one of the two symbols and then waits for the next symbol.

f) In this case, the next symbol is again the same, i.e. B. The receiver, after performing the required calculations among the stored symbol and third symbol, comes to a conclusion that the third symbol is also same as the stored symbol and thus, first three symbols are all the same

g) Now, it waits for the fourth symbol, which is distinct, i.e. A in this case. By performing calculations and with help of the table, the receiver is able to detect that the stored symbol and the fourth symbol are B and A respectively.

h) The receiver now knows that the symbols sent till now were B B B A (as it knew that the first three symbols are same). 
i) After this, it stores the first two distinct symbols, i.e. B and A in this case. Among these, it has already stored B, so it will now store A.

j) Once, it has stored two distinct symbols, it can now detect any symbols, even if two same symbols are encountered one after the other.

k) We need two distinct symbols, and not one symbol, because there might be a case where the received symbol is same as the stored symbol. For example, in this case, had the receiver stored only one symbol, i.e. $\mathrm{B}$ and the next received symbol is also a $\mathrm{B}$, then it would again face the problem of not being able to detect two consecutive same symbols correctly.

1) However, since we have a second distinct stored symbol, it comes into play when the first stored symbol and the received symbol are same.

Table 1. Detection criteria at the receiver for 2 phase weights

\begin{tabular}{|c|c|c|c|c|}
\hline $\begin{array}{l}\text { Symbol sent by the transmitter } \\
\qquad\left(a_{1}, b_{1}\right),\left(a_{2}, b_{2}\right)\end{array}$ & \multicolumn{4}{|c|}{ Equations to detect the symbols } \\
\hline $\mathrm{A}, \mathrm{A}$ & $\left|a_{2}\right|-\left|a_{1}\right|$ & $=0$ & $\left|b_{2}\right|-\left|b_{1}\right|=$ & \\
\hline $\mathrm{A}, \mathrm{B}$ & $\left|a_{2}\right|-\left|2 a_{1}\right|$ & $=0$ & $\left|b_{2}\right|-\left|3 b_{1}\right|=$ & $=0$ \\
\hline $\mathrm{A}, \mathrm{C}$ & $\left|a_{2}\right|-\left|4 a_{1}\right|$ & $=0$ & $\left|b_{2}\right|-\left|2 b_{1}\right|=$ & $=0$ \\
\hline A,D & $\left|a_{2}\right|-\left|5 a_{1}\right|$ & $=0$ & $\left|b_{2}\right|-\left|4 b_{1}\right|=$ & $=0$ \\
\hline B, B & $\left|a_{2}\right|-\left|a_{1}\right|$ & $=0$ & $\left|b_{2}\right|-\left|b_{1}\right|=$ & \\
\hline B, A & $\left|a_{2}\right|-\left|\frac{1}{2} a_{1}\right|$ & $=0$ & $\left|b_{2}\right|-\left|\frac{1}{3} b_{1}\right|$ & $=0$ \\
\hline B,C & $\left|a_{2}\right|-\left|2 a_{1}\right|$ & & $\left|b_{2}\right|-\left|\frac{2}{3} b_{1}\right|$ & $=0$ \\
\hline B, D & $\left|a_{2}\right|-\left|\frac{5}{2} a_{1}\right|$ & $=0$ & $\left|b_{2}\right|-\left|\frac{4}{3} b_{1}\right|$ & $=0$ \\
\hline $\mathrm{C}, \mathrm{C}$ & $\left|a_{2}\right|-\left|a_{1}\right|$ & $=0$ & $\left|b_{2}\right|-\left|b_{1}\right|=$ & \\
\hline $\mathrm{C}, \mathrm{A}$ & $\left|a_{2}\right|-\left|\frac{1}{4} a_{1}\right|$ & $=0$ & $\left|b_{2}\right|-\mid \frac{1}{2} b_{1}$ & $=0$ \\
\hline C, B & $\left|a_{2}\right|-\left|\frac{1}{2} a_{1}\right|$ & $=0$ & $\left|b_{2}\right|-\left|\frac{3}{2} b_{1}\right|$ & $=0$ \\
\hline C, D & $\left|a_{2}\right|-\left|\frac{5}{4} a_{1}\right|$ & $=0$ & $\left|b_{2}\right|-\left|2 b_{1}\right|$ & \\
\hline $\mathrm{D}, \mathrm{D}$ & $\left|a_{2}\right|-\left|a_{1}\right|$ & $=0$ & $\left|b_{2}\right|-\left|b_{1}\right|=$ & \\
\hline $\mathrm{D}, \mathrm{A}$ & $\left|a_{2}\right|-\left|\frac{1}{5} a_{1}\right|$ & $=0$ & $\left|b_{2}\right|-\left|\frac{1}{4} b_{1}\right|$ & $=0$ \\
\hline D, B & $\left|a_{2}\right|-\left|\frac{2}{5} a_{1}\right|$ & $=0$ & $\left|b_{2}\right|-\mid \frac{3}{4} b_{1}$ & $=0$ \\
\hline $\mathrm{D}, \mathrm{C}$ & $\left|a_{2}\right|-\left|\frac{4}{5} a_{1}\right|$ & $=0$ & $\left|b_{2}\right|-\mid \frac{1}{2} b_{1}$ & $=0$ \\
\hline
\end{tabular}


So the receiver uses the two consecutive received symbols for detection. However, when the two received symbols are same, it uses one of the stored symbols to complete the detection without any problem. The rest of the detection process continues in a similar way.

7. An advantage of using the proposed method is that we can find out which symbols were transmitted even if they have been multiplied by different phase factors

8. For example : Suppose we choose phase factors $\{ \pm 1\}$, then let a symbol that is multiplied by phase factor -1 be represented as [ ]'

So, if we want to transmit A B where A is multiplied by phase factor 1 and B by -1 then it is represented as A B' and so on and so forth. The receiver can detect A B even if A B or A' B or A B' or A' B' was transmitted which is good because it doesn't require any side information to detect the symbols correctly.

The same (that is discussed in point vii.) is true even if we choose $\{ \pm j\}$ as phase factors instead of $\{ \pm 1\}$ but in that case the equations (at the receiver) of real and imaginary part would interchange. For example, if in case of $(+1,-1)$, the equations were

$$
\left|a_{2}\right|-\left|4 a_{1}\right|=0 \quad\left|b_{2}\right|-\left|4 b_{1}\right|=0
$$

Then in case of $\{ \pm j\}$, the equations would be

$$
\left|b_{2}\right|-\left|4 b_{1}\right|=0 \quad\left|a_{2}\right|-\left|2 a_{1}\right|=0
$$

\section{B. The Proposed method with 4- phase weights}

We can also use 4 phase factors together i.e. $\{ \pm 1, \pm j\}$ but in that case, keeping in mind the complexity of the receiver, we will have to add a constraint to the use of these phase factors Let us suppose that $\{ \pm 1\}$ is Group 1 and $\{ \pm j\}$ is Group 2.The constraint is that, we will have to multiply the two symbols (that have to be transmitted one after another) by the same phase group i.e. either group 1 or group 2.

For example, if we have to transmit a sequence $\mathrm{B}-\mathrm{C}-\mathrm{A}-\mathrm{D}$, then we know that the transmitter would send two symbols simultaneously and the constraint is that both the symbols $\mathrm{B}$ and $\mathrm{C}$ would have to be multiplied by the same phase group i.e. either group 1 or group 2 . However, when transmitting the next two symbols i.e. A and D (in this case), we can multiply these by a different group than previous one (if we want to). Also, in this case, the problem in detection on receiving two same consecutive symbols (A A, B B, C C or D D) has to be solved with the help of Euclidean distance as discussed in Part 1 . The set of equations to identify the symbols would also be more than in case of two phase factors. The equations for points A, B, $\mathrm{C}$ and $\mathrm{D}$ are shown in Table 2. In this case, even if one set of equations get satisfied, the receiver would detect that the corresponding symbols that were sent.

As we know that for $O$ phase weights and $U$ sub-blocks in PTS the number of side information bits is given by:

Number of SI bits in conventional PTS $=(U-1) \cdot \log _{2} O$

Table 3 represents the number of SI bits required per OFDM symbol in PTS for different values of phase weights and sub-block sizes. This represents the number of bandwidth saved in terms of bits per OFDM symbol in our proposed scheme as the proposed scheme does not require SI bits.

As far as degradation in performance of the receiver is concerned it is always better than conventional ML method typically used in PTS methods as the proposed method is much simpler than ML method. 
Alok Joshi, et al.

Table 2. Detection criteria at the receiver for 2 phase weights for 4 phase weights

\begin{tabular}{|c|c|c|c|}
\hline $\begin{array}{l}\text { Symbol sent by the } \\
\text { transmitter }\left(a_{1}, b_{1}\right) \text {, } \\
\qquad\left(a_{2}, b_{2}\right)\end{array}$ & $\begin{array}{l}\text { Equations to detect the symbols( } \\
\text { set-1) }\end{array}$ & Equations to detect the & symbols (set-2) \\
\hline $\mathrm{A}, \mathrm{A}$ & $\begin{array}{l}\left|a_{2}\right|-\left|a_{1}\right|=0 \\
\left|b_{2}\right|-\left|b_{1}\right|=0\end{array}$ & $\left|a_{2}\right|-\left|a_{1}\right|=0$ & $\left|b_{2}\right|-\left|b_{1}\right|=0$ \\
\hline $\mathrm{A}, \mathrm{B}$ & $\begin{array}{l}\left|a_{2}\right|-\left|2 a_{1}\right|=0 \\
\left|b_{2}\right|-\left|3 b_{1}\right|=0\end{array}$ & $\begin{array}{r}\left|b_{2}\right|-\left|2 b_{1}\right| \\
\left|a_{2}\right|-\left|3 a_{1}\right| \\
\end{array}$ & $\begin{array}{l}\mid=0 \\
\mid=0\end{array}$ \\
\hline $\mathrm{A}, \mathrm{C}$ & $\begin{array}{l}\left|a_{2}\right|-\left|4 a_{1}\right|=0 \\
\left|b_{2}\right|-\left|2 b_{1}\right|=0\end{array}$ & $\left|b_{2}\right|-\left|4 b_{1}\right|=0$ & $\left|a_{2}\right|-\left|2 a_{1}\right|=0$ \\
\hline A,D & $\begin{array}{l}\left|a_{2}\right|-\left|5 a_{1}\right|=0 \\
\left|b_{2}\right|-\left|4 b_{1}\right|=0\end{array}$ & $\left|b_{2}\right|-\left|5 b_{1}\right|=0$ & $\left|a_{2}\right|-\left|4 a_{1}\right|=0$ \\
\hline B, B & $\begin{array}{l}\left|a_{2}\right|-\left|a_{1}\right|=0 \\
\left|b_{2}\right|-\left|b_{1}\right|=0\end{array}$ & $\left|b_{2}\right|-\left|b_{1}\right|=0$ & $\left|a_{2}\right|-\left|a_{1}\right|=0$ \\
\hline $\mathrm{B}, \mathrm{A}$ & $\begin{array}{l}\left|a_{2}\right|-\left|\frac{1}{2} a_{1}\right|=0 \\
\left|b_{2}\right|-\left|\frac{1}{3} b_{1}\right|=0\end{array}$ & $\begin{array}{l}\left|b_{2}\right|-\mid \frac{1}{2} b_{1} \\
\left|a_{2}\right|-\mid \frac{1}{3} a_{1}\end{array}$ & $\begin{array}{l}\mid=0 \\
1=0\end{array}$ \\
\hline $\mathrm{B}, \mathrm{C}$ & $\begin{array}{l}\left|a_{2}\right|-\left|2 a_{1}\right|=0 \\
\left|b_{2}\right|-\left|\frac{2}{3} b_{1}\right|=0\end{array}$ & $\begin{array}{l}\left|b_{2}\right|-\left|2 b_{1}\right| \\
\left|a_{2}\right|-\mid \frac{2}{3} a_{1}\end{array}$ & $\begin{array}{l}\mid=0 \\
1=0\end{array}$ \\
\hline $\mathrm{B}, \mathrm{D}$ & $\begin{array}{l}\left|a_{2}\right|-\left|\frac{5}{2} a_{1}\right|=0 \\
\left|b_{2}\right|-\left|\frac{4}{3} b_{1}\right|=0\end{array}$ & $\begin{array}{l}\left|b_{2}\right|-\left|\frac{5}{2} b_{1}\right| \\
\left|a_{2}\right|-\mid \frac{4}{3} a_{1}\end{array}$ & $\begin{array}{l}=0 \\
=0\end{array}$ \\
\hline $\mathrm{C}, \mathrm{C}$ & $\begin{array}{l}\left|a_{2}\right|-\left|a_{1}\right|=0 \\
\left|b_{2}\right|-\left|b_{1}\right|=0\end{array}$ & $\left|a_{2}\right|-\left|a_{1}\right|=0$ & $\left|b_{2}\right|-\left|b_{1}\right|=0$ \\
\hline $\mathrm{C}, \mathrm{A}$ & $\begin{array}{l}\left|a_{2}\right|-\left|\frac{1}{4} a_{1}\right|=0 \\
\left|b_{2}\right|-\left|\frac{1}{2} b_{1}\right|=0\end{array}$ & $\begin{array}{l}\left|b_{2}\right|-\left|\frac{1}{4} b_{1}\right| \\
\left|a_{2}\right|-\mid \frac{1}{2} a_{1}\end{array}$ & $\begin{array}{l}=0 \\
1=0\end{array}$ \\
\hline $\mathrm{C}, \mathrm{B}$ & $\begin{array}{l}\left|a_{2}\right|-\left|\frac{1}{2} a_{1}\right|=0 \\
\left|b_{2}\right|-\left|\frac{3}{2} b_{1}\right|=0\end{array}$ & $\begin{array}{r}\left|b_{2}\right|-\left|\frac{1}{2} b_{1}\right| \\
\left|a_{2}\right|-\mid \frac{3}{2} a_{1}\end{array}$ & $\begin{array}{l}\mid=0 \\
1=0\end{array}$ \\
\hline
\end{tabular}




\begin{tabular}{|c|c|c|}
\hline C, D & $\left|a_{2}\right|-\left|\frac{5}{4} a_{1}\right|=0$ & $\left|b_{2}\right|-\left|\frac{5}{4} b_{1}\right|=0$ \\
\hline$b_{2}|-| 2 b_{1} \mid=0$ & $\left|a_{2}\right|-\left|2 a_{1}\right|=0$ \\
\hline D, D & $\left|a_{2}\right|-\left|a_{1}\right|=0$ & $\left|a_{2}\right|-\left|a_{1}\right|=0$ \\
\hline$b_{2}|-| b_{1} \mid=0$ & $\left|b_{2}\right|-\left|\frac{1}{5} b_{1}\right|=0$ \\
\hline D, A & $\left|a_{2}\right|-\left|\frac{1}{5} a_{1}\right|=0$ & $\left|a_{2}\right|-\left|\frac{1}{4} a_{1}\right|=0$ \\
\hline D, B & $\left|b_{2}\right|-\left|\frac{1}{4} b_{1}\right|=0$ & $\left|b_{2}\right|-\left|\frac{2}{5} b_{1}\right|=0$ \\
\hline$a_{2}|-| \frac{2}{5} a_{1} \mid=0$ & $\left|a_{2}\right|-\left|\frac{3}{4} a_{1}\right|=0$ \\
\hline D, C & $\left|b_{2}\right|-\left|\frac{3}{4} b_{1}\right|=0$ & $\left|b_{2}\right|-\left|\frac{4}{5} b_{1}\right|=0$ \\
\hline$a_{2}|-| \frac{4}{5} a_{1} \mid=0$ & $\left|a_{2}\right|-\left|\frac{1}{2} a_{1}\right|=0$ \\
\hline
\end{tabular}

Table 3. Number of side bits required for transmission per OFDM symbol

\begin{tabular}{|c|c|c|}
\hline $\begin{array}{c}\text { Number of phase } \\
\text { weights }(O)\end{array}$ & $\begin{array}{c}\text { Number of sub-blocks } \\
(U-1)\end{array}$ & $\begin{array}{c}\text { Side information bits } \\
\text { in Conventional PTS } \\
(U-1) \cdot \log _{2} O\end{array}$ \\
\hline 2 & 4 & 3 \\
\hline 4 & 6 & 10 \\
\hline 8 & 8 & 21 \\
\hline
\end{tabular}

\section{Simulation results}

The simulations are done for 10000 OFDM symbols with 64 FFT size and 16 QAM mapping. For PTS block sizes of 2, 4, 6 sub blocks The PAPR values are tabulated in Table 4. Table 4. Values for PAPR in $\mathrm{dB}$

\begin{tabular}{|c|c|c|c|}
\hline \multirow{2}{*}{$\begin{array}{c}\text { Sub-block Size } \\
(\mathrm{M})\end{array}$} & \multicolumn{3}{|c|}{ PAPR (dB) } \\
\cline { 2 - 3 } & Plain PTS & Proposed PTS & OFDM \\
\hline 4 & 5.75 & 5.80 & \multirow{2}{*}{9.23} \\
\hline 6 & 4.80 & 4.90 & \\
\hline 8 & 3.8 & 3.78 & \\
\hline
\end{tabular}

As far as PAPR is concerned, there is no degradation in PAPR reduction capability of PTS method with proposed modifications as seen by overlapping curves in Figure 3.

PAPR values clearly suggest that plain PTS and proposed PTS have similar PAPR performance for various values of sub block sizes. 
Alok Joshi, et al.

Tables also show that PTS drastically reduce the value of PAPR than conventional OFDM system. So the proposed scheme does not hamper the PAPR reduction capability of PTS method.

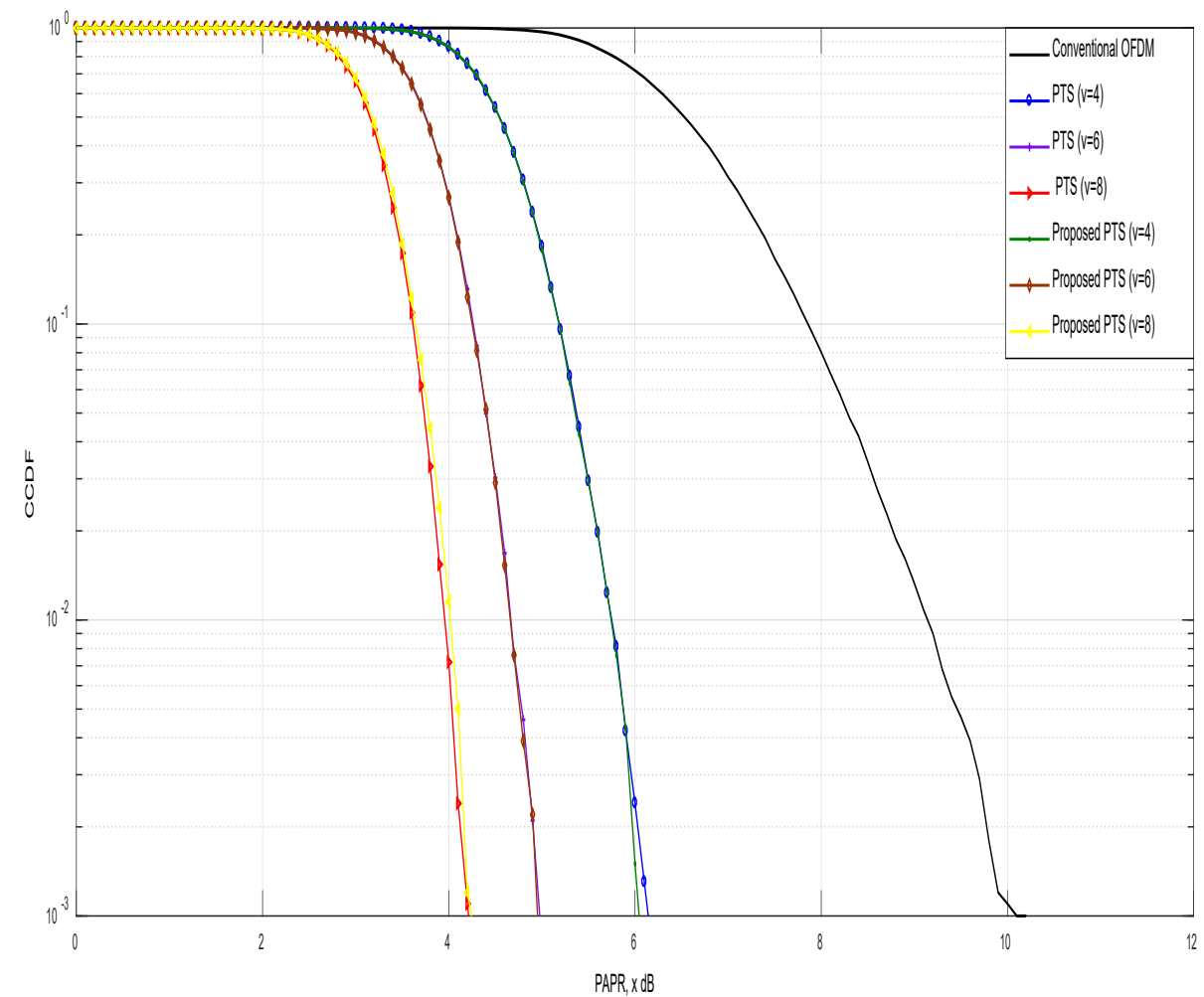

Figure 3. PAPR comparison of conventional OFDM, proposed and plain PTS technique

The PAPR values are well within the range for standard LTE transmission in the downlink. The power requirement of transmission is also not changed which is shown in the power spectral density (PSD) curve in Figure. 4. The PSD curve is drawn for $20 \mathrm{MHZ}$ bandwidth.

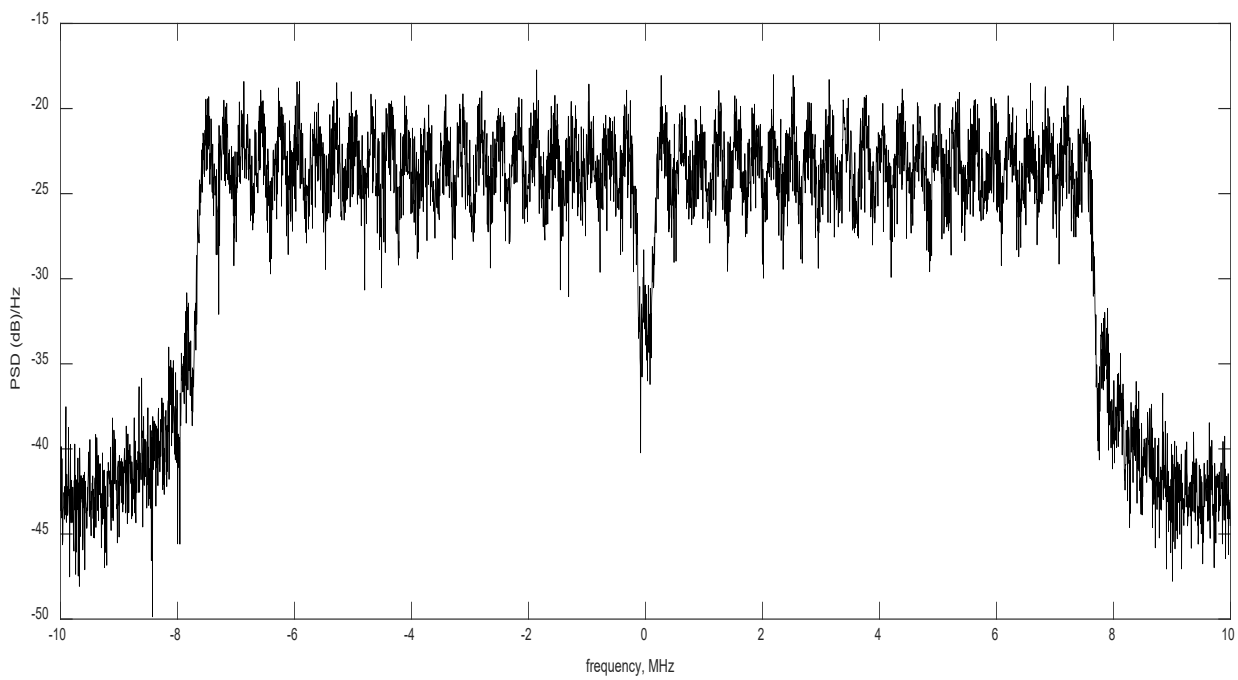

Figure 4. PSD curve for transmitted signal in proposed scheme 


\section{A. Comparison with other proposed methods available in literature}

The proposed scheme avoids the need of SI at the receiver based on verification of few simple mathematical constraints. Most of the methods described in literature use ML method for deciding upon the received data vector. ML method ased receiver involves higher computations and complex structures which increases by size of constellation. In [22] to avoid SI transmission a predetermined number of pilot signals are inserted in each sub-block for SI detection and at the receiver Euclidian distance is calculated to detect the transmitted data vector and it assume the allowable phase factors are known to the receiver which make receiver pretty complexity. Use of the pilot tones also reduces the available bandwidth for the data transmission.

In [23] each sub block is cyclically shifted to introduce the phase offset, at the receiver complex maximum likelihood detector is required to find the shift used at the transmitter and at the same time computation complexity is higher at the receiver.

In [24] a reshaped QAM constellation is used with similar minimum distance and used with PTS at the receiver minimum mean square error criteria is used for data vector detection for each of the rotated modified QAM constellation with received one which is cumbersome process. In [25] too extra pilot symbol is added in each of the sub block to enable decoding the process at the receiver resulting in to lesser net data rate.

In [26] single IFFT operation replaces multiple IFFT operation of a PTS scheme which results in to lesser IFFT computations and at each of the sub block phase rotation and offset are used together and a complex ML receiver with relatively higher computation is used at the receiver due higher and complex mathematical constraints due to joint phase rotation and offset.

In [27] SI free PTS is proposed especially for Alamouti's MIMO transmitter with phase offset and rotation both are used. The computations and complexity are higher due to complex mathematical calculations and ML detection as it is designed for MIMO system.

In [28] wavelet packet based OFDM is used to reduce complexity but uses embedded SI which results in to data rate loss with complex receiver design. In [29] too uses phase offset on rotating vectors and uses embedded SI with ML based receiver. Apart from this scheme also requires rigorous computations in detecting the phase offset at the receiver.

\section{Conclusion}

In this paper, the proposed technique avoids use of explicit side information in PTS. Although the performance in terms of PAPR reduction remains same, the spectral efficiency of the system improves significantly. Even though the proposed scheme works without side information, it only requires relatively less computations than a standard ML based receiver.

\section{References}

[1]. Y. S. Cho, J.Kim, W.Y. Yang, C. G. Kang, "MIMO-OFDM WIRELESS COMMUNICATIONS WITH MATLAB", IEEE press and Willey Publications, pp.111150,2010

[2]. Jha, U.S; Prasad R., "OFDM Towards Fixed and Mobile Broadband Wireless Access", Artech House, Inc. Norwood, MA, USA C 2007

[3]. J. Hao, J. Wang and C. Pan, "Low Complexity ICI Mitigation for MIMO-OFDM in Time-Varying Channels," in IEEE Transactions on Broadcasting, vol. 62, no. 3, pp. 727735, Sept. 2016.

[4]. Y. Rahmatallah and S. Mohan, "Peak-To-Average Power Ratio Reduction in OFDM Systems: A Survey And Taxonomy," in IEEE Communications Surveys \& Tutorials, vol. 15, no. 4, pp. 1567-1592, Fourth Quarter 2013.

[5]. R. M. Ali, R. K. Rao and V. Parsa, "PAPR reduction in OFDM systems using clipping based on symbol statistics," 2017 IEEE 30th Canadian Conference on Electrical and Computer Engineering (CCECE), Windsor, ON, 2017 
[6]. K. Anoh, C. Tanriover and B. Adebisi, "On the Optimization of Iterative Clipping and Filtering for PAPR Reduction in OFDM Systems," in IEEE Access, vol. 5, no., pp. 12004-12013, 2017.

[7]. Sohn and S. C. Kim, "Neural Network Based Simplified Clipping and Filtering Technique for PAPR Reduction of OFDM Signals," in IEEE Communications Letters, vol. 19, no. 8, pp. 1438-1441, Aug. 2015.

[8]. S. Cha, M. Park, S. Lee, K. J. Bang and D. Hong, "A new PAPR reduction technique for OFDM systems using advanced peak windowing method," in IEEE Transactions on Consumer Electronics, vol. 54, no. 2, pp. 405-410, May 2008

[9]. N. Ali, R. Almahainy, A. Al-Shabili, N. Almoosa and R. Abd-Alhameed, "Analysis of mproved $\mu$-law companding technique for OFDM systems," in IEEE Transactions on Consumer Electronics, vol. 63, no. 2, pp. 126-134, May 2017.

[10]. H. B. Jeon, J. S. No and D. J. Shin, "A New PAPR Reduction Scheme Using Efficient Peak Cancellation for OFDM Systems," in IEEE Transactions on Broadcasting, vol. 58, no. 4, pp. 619-628, Dec. 2012.

[11]. G. Bai, Z. Zhong, R. Xu, G. Wang and Z. Qin, "Golay complementary sequences and Reed-Muller codes based PAPR reduction for relay networks with superimposed training," 2012 IEEE 11th International Conference on Signal Processing, Beijing, 2012, pp. $1558-1561$.

[12]. O. Daoud and O. Alani, "Reducing the PAPR by utilisation of the LDPC code," in IET Communications, vol. 3, no. 4, pp. 520-529, April 2009.

[13]. M. Sabbaghian, Y. Kwak, B. Smida and V. Tarokh, "Near Shannon Limit and Low Peak to Average Power Ratio Turbo Block Coded OFDM," in IEEE Transactions on Communications, vol. 59, no. 8, pp. 2042-2045, August 2011.

[14]. W. Wang, M. Hu, Y. Li and H. Zhang, "A Low-Complexity Tone Injection Scheme Based on Distortion Signals for PAPR Reduction in OFDM Systems," in IEEE Transactions on Broadcasting, vol. 62, no. 4, pp. 948-956, Dec. 2016.

[15]. C. Ni, Y. Ma and T. Jiang, "A Novel Adaptive Tone Reservation Scheme for PAPR Reduction in Large-Scale Multi-User MIMO-OFDM Systems," in IEEE Wireless Communications Letters, vol. 5, no. 5, pp. 480-483, Oct. 2016.

[16]. J. Zhong, X. Yang and W. Hu, "Performance-Improved Secure OFDM Transmission Using Chaotic Active Constellation Extension," in IEEE Photonics Technology Letters, vol. 29, no. 12, pp. 991-994, June15, 152017.

[17]. R. W. Bauml, R. F. H. Fischer, and J. B. Huber, "Reducing the peak-to-average power ratio of multicarrier modulation by selected mapping," Electron. Lett., vol. 32, no. 22, pp. 2056-2057, Oct. 1996.

[18]. N. Taşpınar and M. Yıldırım, "A Novel Parallel Artificial Bee Colony Algorithm and Its PAPR Reduction Performance Using SLM Scheme in OFDM and MIMO-OFDM Systems," in IEEE Communications Letters, vol. 19, no. 10, pp. 1830-1833, Oct. 2015.

[19]. S. H. Wang, K. C. Lee and C. P. Li, "A Low-Complexity Architecture for PAPR Reduction in OFDM Systems with Near-Optimal Performance," in IEEE Transactions on Vehicular Technology, vol. 65, no. 1, pp. 169-179, Jan. 2016.

[20]. L. J. Cimini and N. R. Sollenberger, "Peak-to-average power ratio reduction of an OFDM signal using partial transmit sequences," in IEEE Communications Letters, vol. 4, no. 3, pp. 86-88, March 2000

[21]. Y. J. Cho, K. H. Kim, J. Y. Woo, K. S. Lee, J. S. No and D. J. Shin, "Low-Complexity PTS Schemes Using Dominant Time-Domain Samples in OFDM Systems," in IEEE Transactions on Broadcasting, vol. 63, no. 2, pp. 440-445, June 2017.

[22]. Giannopoulos, Th, \& Paliouras, V. (2009). A low-complexity PTS-based PAPR reduction technique for O OFDM signals without transmission of side information. Springer Journal of Signal Processing Systems, 56(2-3), 141-153. 
[23]. L. Yang, K. K. Soo, S. Q. Li and Y. M. Siu, "PAPR Reduction Using Low Complexity PTS to Construct of OFDM Signals Without Side Information," in IEEE Transactions on Broadcasting, vol. 57, no. 2, pp. 284-290, June 2011.

[24]. C. Li, T. Jiang, Y. Zhou and H. Li, "A Novel Constellation Reshaping Method for PAPR Reduction of OFDM Signals," in IEEE Transactions on Signal Processing, vol. 59, no. 6, pp. 2710-2719, June 2011

[25]. H. Kim, E. Hong, C. Ahn and D. Har, "A Pilot Symbol Pattern Enabling Data Recovery Without Side Information in PTS-Based OFDM Systems," in IEEE Transactions on Broadcasting, vol. 57, no. 2, pp. 307-312, June 2011.

[26]. S. S. Eom, H. Nam and Y. C. Ko, "Low-Complexity PAPR Reduction Scheme Without Side Information for OFDM Systems," in IEEE Transactions on Signal Processing, vol. 60, no. 7, pp. 3657-3669, July 2012.

[27]. T. Jiang, C. Ni, L. Guan and Q. Qi, "Peak-to-average power ratio reduction in alamouti multi-input-multi-output orthogonal frequency division multiplexing systems without side information using phase offset based-partial transmit sequence scheme," in IET Communications, vol. 8, no. 5, pp. 564-570, March 272014.

[28]. J. Zakaria and M. F. Mohd Salleh, "PAPR reduction scheme: wavelet packet-based PTS with embedded side information data scheme," in IET Communications, vol. 11, no. 1, pp. 127-135, 152017.

[29]. H. S. Joo, K. H. Kim, J. S. No and D. J. Shin, "New PTS Schemes for PAPR Reduction of OFDM Signals Without Side Information," in IEEE Transactions on Broadcasting, vol. 63, no. 3, pp. 562-570, Sept. 2017.
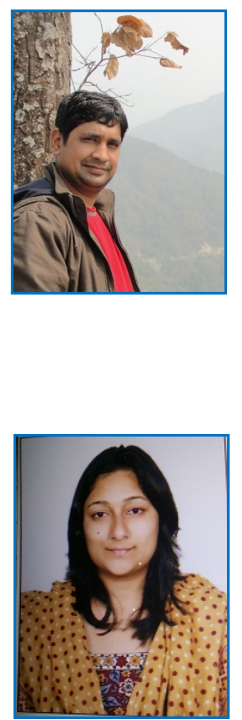

Alok Joshi received B.E degree in Electronics \& Communication Engg. from G.B. Pant Engg. College (H.N.B, Garhwal University) Uttarakhand, India in 2001 and M.Tech in Digital Communication from U.P.T.U Lucknow (India) in 2006. Received PhD degree from JUIT in 2014, in the area of wireless communications. He has total teaching experience of 19 years in various technical universities in India. Currently he is working as Associate professor at Jaypee Institute of Information Technology, Noida, India. His research interests are wireless systems.

Samriti Kalia received B.E degree in Electronics \& Communication Engg. from G.T.B.K.I.E.T Malout, Punjab Technical Univ, Jalandhar, (India) in 2003 and M.Tech in Electronic Product Design and Technology, Punjab Engineering College, Deemed Univ, Chandigarh, (India) in 2006. Currently she is pursuing $\mathrm{PhD}$ degree from Jaypee Institute of Information Technology, Noida (India) in the area of wireless communications. She has total teaching experience of 7 years. Her interest areas are MIMO, OFDM systems, fading models. 